\title{
Kalman filter observation error model applied to vehicle tracking dynamic obstacle correction
}

\author{
S. M. Siao ${ }^{1 *}$, Y. R. Chen ${ }^{2}$, L. Y. Shu ${ }^{3}$ \\ 1, 2, 3 Automotive Research \& Testing Center, Changhua, Taiwan
}

\section{Keywords \\ ADAS \\ Kalman filter \\ Radar}

Received: 3 April 2019

Accepted: 6 May 2019

Published: 28 June 2019

\begin{abstract}
To increase accuracy for dynamic detection obstacles, this paper presents a Kalman filter observation error model based on $77 \mathrm{GHz}$ middle range radar (MRR). In recently, the vehicle equips advanced driver assistance system (ADAS), which becomes more popular. Among these, the accuracy of the obstacle information, such as distance, relative speed, and position, is the most important purpose. However, radar is one of the main detection sensors, but its data transmission delay, including millimeter-wave reflection, the analogy to digital signal conversion, and data process, would influence radar tracking correction. Therefore, algorithm of the proposed model adopts detection time and a dynamic estimated model of an obstacle to compensate detection delay, such that the dynamic correction means error can be reduced. Next, the testing condition sets RTK-GPS as the real-world reference frame, the experimental would be realized with the dynamic scene. According to the above results, when the target distance closer 100 meter, our model's dynamic correction mean error was improved 59\%, 74\%, 78\% during in 20kph, $40 \mathrm{kph}$, and $60 \mathrm{kph}$ relatively speed, respectively.
\end{abstract}

(C) 2019 The Author(s). Published by TAF Publishing.

\section{INTRODUCTION}

In recent years, many automobile factories have been investing in the ADAS [1] development for manufacturing the modern safety car. It is assembled with several systems, such as Adaptive Cruise Control (ACC), Lane-Keeping Assistance (LKA), Autonomous Emergency Braking (AEB), Lane Change Assistance (LCA), and so on. According to the Society of Automotive Engineers (SAE) specification, this intelligent system is called partial automation which simultaneously integrates more than one body control into executing assistance operation based on the driving environment, but the driver is still mainly observer so that it is classified as the second level. For precise control, the obstacle detection of the environment is the most important information. Therefore, the sensor which has detection range function, such as ultrasonic, camera, lidar, and millimeter-wave radar, is developed. However, the features of these sensors are different. For example, ultrasonic is a low-cost sensor, but it does not appropriately detect long-range obstacles, which are longer than 5 meters. Next, the camera not only can detect over 50 meters but also cognate the type of obstacle. Nevertheless, its detection ability is affected by night. Then, the three-dimensional lidar can be operated day and night, but its cost is higher than the other sensors. Relatively, although millimeter-wave radar could not cognate the obstacle, it has some advantages which include longrange detection and all-day operation time. Besides, it can obtain more precise relative speed about obstacles than the other sensors based on Frequency Modulated Continuous Wave (FMCW) theorem. Therefore, it is one of the appropriate sensors which is responsible for long-range detection, tracking, and prediction of obstacle movement in ADAS $[2,3,4,5,6,7,8,9,10,11]$.

Under the radar operating process, the antenna is used to transmit millimeter waves and then receive a reflection signal of obstacles. Next, the difference in time of the signal back and forth is used to compute the obstacles information which includes relative speed, distance, and angle. Among these, during the period of radar sensing time, the latency delay would be generated $[12,13,14]$, and a part of obstacle

\footnotetext{
${ }^{*}$ Corresponding author: S. M. Siao

†email: marksiao@artc.org.tw
} 
information could be lost during this delay time. It causes that AEB system tracking precisely would be reduced. For example, each 0.1-second delay makes that any object information can not be provided to the system while moving 1.67 meters when a vehicle is driving at a speed of 60 kilometers per hour. Besides, the signal point would generate drifting or missing by environment interference $[15,16]$. Therefore, the development of a reliable compensate algorithm for rebuilding lost data is the most important issue.

Based on the Kalman filter observation error model, a new dynamic obstacle correction algorithm that is used to improve radar tracking dynamic vehicle performance is proposed in this paper. First, for filtering input data, the observation error model of the obstacle is designed based on Kalman's theory $[15,17,18]$. Next, we analyze several detection information pieces, such as the location and dynamics of obstacle and detection time. According to the above filtering and analysis results, we can compute the compensation time of the detection of obstacle requirements and then the detection accuracy of dynamic obstacles could be improved. From experimental results, the AEB system adopts the proposed method; the dynamic correction mean error of our model was improved by $59 \%, 74 \%$, and $78 \%$ in $20 \mathrm{kph}, 40 \mathrm{kph}$, and $60 \mathrm{kph}$ relative speed when the distance of subject was closer than 100 meters, respectively.

The remainder of this paper is organized as follows: Section 2 shows the derivation method of the Kalman filter and the observation error model. Next, the procedure of detection obstacle time analysis of the proposed architecture and its dynamic correction method is introduced in Section 3. Finally, Section 4 presents the conclusions of this study.

\section{A. Kalman Filter and Observation Error Model}

Equations 1, 2, 3, 4, 5 are the equations for the Kalman filter. The estimated value for the previous point and the observation value for the current point are used to calculate the estimated value of the minimum mean square error [19]. Observation error models with various distances are provided to make the output close to the real state. Details are provided in the following:

$$
\hat{\mathbf{x}}_{k \mid k-1}=A \hat{\mathbf{x}}_{k-1 \mid k-1}+B \mathbf{u}_{k-1}
$$

$$
\begin{gathered}
P_{k \mid k-1}=A P_{k-1 \mid k-1} A^{T}+Q \\
K_{k}=P_{k k-1} H^{T}\left(H P_{k k-1} H^{T}+R\right)^{-1} \\
\hat{\mathbf{x}}_{k \mid k}=\hat{\mathbf{x}}_{k \mid k-1}+K_{k}\left(Z_{\mathrm{k}}-H \hat{\mathbf{x}}_{k \mid k-1}\right) \\
P_{k \mid k}=\left(I-K_{k} H\right) P_{k \mid k-1}
\end{gathered}
$$

Considering Equation 1 for the Kalman filter, the state of the previous point $\left(\mathbf{x}_{k-1 \mid k-1}\right)$ is first used to estimate the current state $\left(\hat{\mathbf{x}}_{k \mid k-1}\right)$, where $A$ is the state-transition matrix specified in Equation 6, $B$ is a control matrix, and $u$ is the control amount.

$$
A=\left[\begin{array}{cccc}
1 & \Delta t & 0 & 0 \\
0 & 1 & 0 & 0 \\
0 & 0 & 1 & \Delta t \\
0 & 0 & 0 & 1
\end{array}\right]
$$

In Equation 2, $P_{k \mid k-1}$ is the covariance matrix of system error moving from the previous point to the current point, $Q$ is system noise, and $A^{T}$ is the transpose of $A$.

Equation 3 calculates the Kalman constant $K$, which can be used to minimize the covariance matrix of system error $P_{k \mid k}$ in Equation 5. Concurrently, $K$ also serves to compute the estimate of the current state $\hat{\mathbf{x}}_{k k}$ and regulates the weights for the observation value in Equation 4. That is, when $K$ is large, the system believes that the observed value is greater than the estimated value; when $K$ is small, the system believes that the estimated value is greater than the observation. Additionally, $H=\left[\begin{array}{llll}1 & 0 & 0 & 0 \\ 0 & 0 & 1 & 0\end{array}\right]$, and $R$ is the matrix of observation error. The covariance matrix of observation value $R$ is a parameter in Equation 3 for adjusting the Kalman constant $K$. A dynamic observation error model can be established by analyzing observation error values for various distances based on radar detection results (e.g., Figure 1). By adjusting the matrix of observation error in Equation 3 the more accurate value of the state $\hat{\mathbf{x}}_{k k}$ in Equation 4 and the smaller covariance matrix of system error $P_{k \mid k}$ can be obtained. 


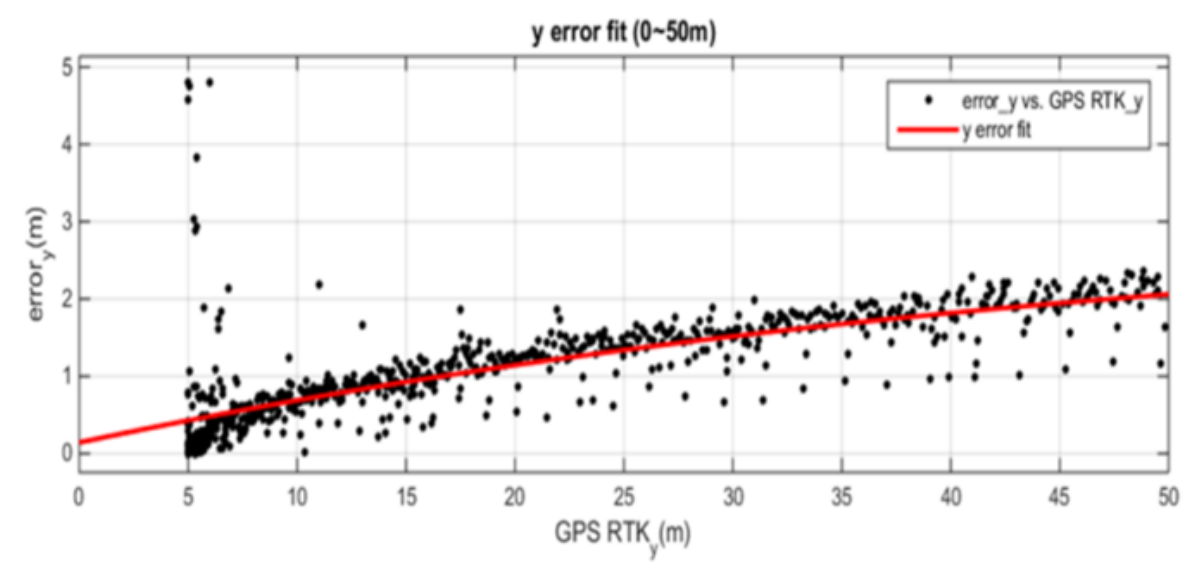

Fig. 1. Analysis of radar error observation at different distances

\section{B. Millimeter-Wave Radar Data Collection}

Table 1 presents the basic performance of the millimeterwave radar used in this study. Two detection ranges of the radar were used: short distance ( $60 \mathrm{~m}$ or below) and long distance (200 m or below; Figure 2). GPS Real-Time Kinematic (GPS-RTK) was used as the real coordinate reference. GPS-RTK can be used for real-time positioning with high precision and is, therefore, suitable to serve as the ground truth. The data collection location was an open area. Within the radar detection range, no other obstacles were found except for the target vehicle. GPS-RTK was installed in the target vehicle (Figure 3). The millimeter-wave radar was set at a fixed position and as the origin. The target vehicle was located $5 \mathrm{~m}$ in front of the radar and began to accelerate and drive forward at a fixed speed. GPS-RTK data and millimeter-wave radar data were simultaneously collected. Some data are presented in Figure 4 and 5.

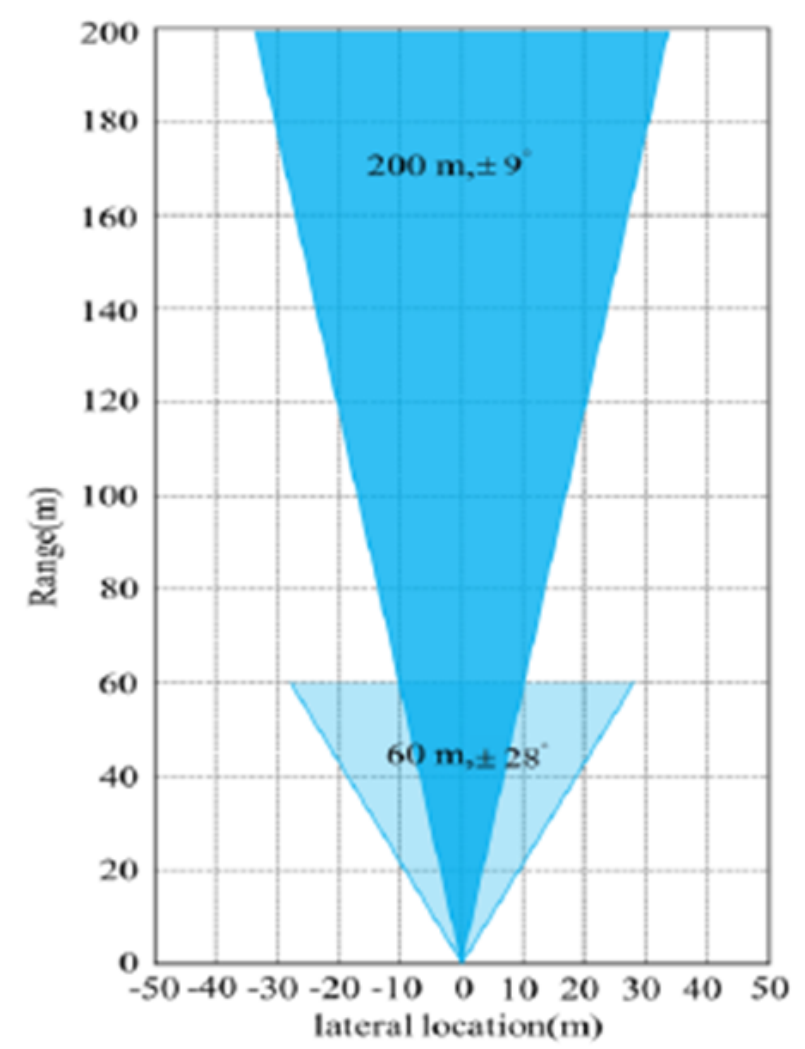

Fig. 2. Field of view of Radar detection range 
TABLE 1

77GHZ RADAR SPECIFICATIONS

\begin{tabular}{|c|c|c|c|c|c|c|}
\hline $\begin{array}{l}\text { Longest } \\
\text { Detection } \\
\text { Range }\end{array}$ & $\begin{array}{l}\text { Nearest } \\
\text { Detection } \\
\text { Range }\end{array}$ & Field of View & Speed Range & $\begin{array}{l}\text { Accuracy } \\
\text { Distance } \\
\text { Measuring }\end{array}$ & $\begin{array}{l}\text { Speed } \\
\text { Accuracy }\end{array}$ & Cycle Time \\
\hline $200 \mathrm{~m}$ & $0.25 \mathrm{~m}$ & $\begin{array}{l}\text { Far: } \pm 9^{\circ} \\
\text { Close-up: } \pm 9^{\circ}\end{array}$ & $88 \mathrm{kph} \sim 265 \mathrm{kph}$ & $\begin{array}{l} \pm 0.25 \mathrm{~m} \text { or } \\
1.5 \% @>1 \mathrm{~m}\end{array}$ & $\pm 0.5 \mathrm{kph}$ & $66 \mathrm{mS}$ \\
\hline
\end{tabular}

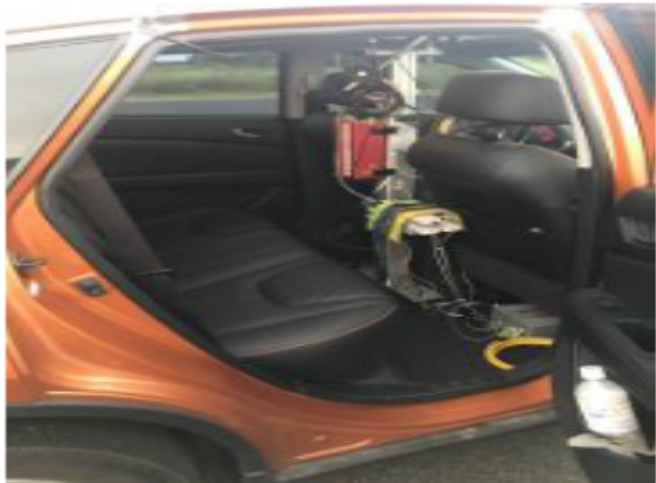

Fig. 3. GPS-RTK installation diagram

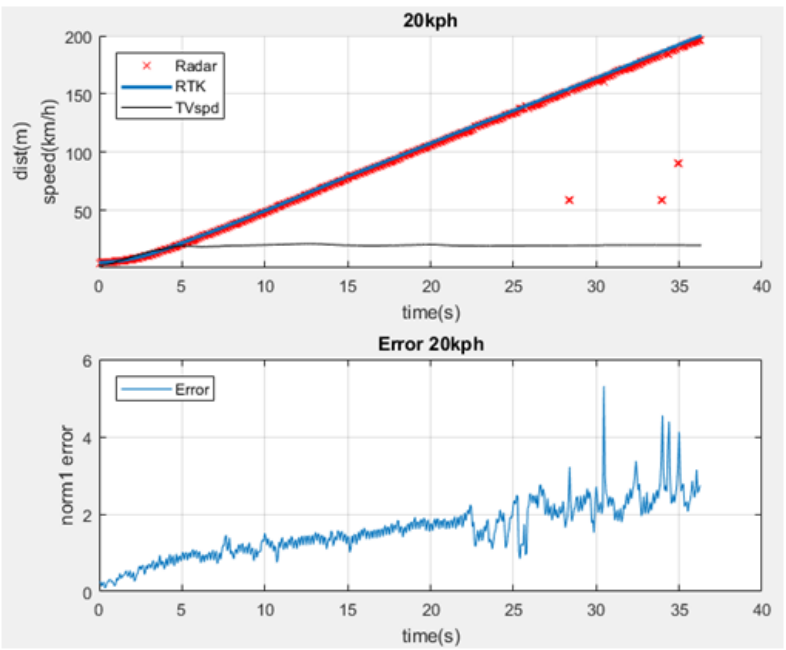

Fig. 4. Data collection of 20kph

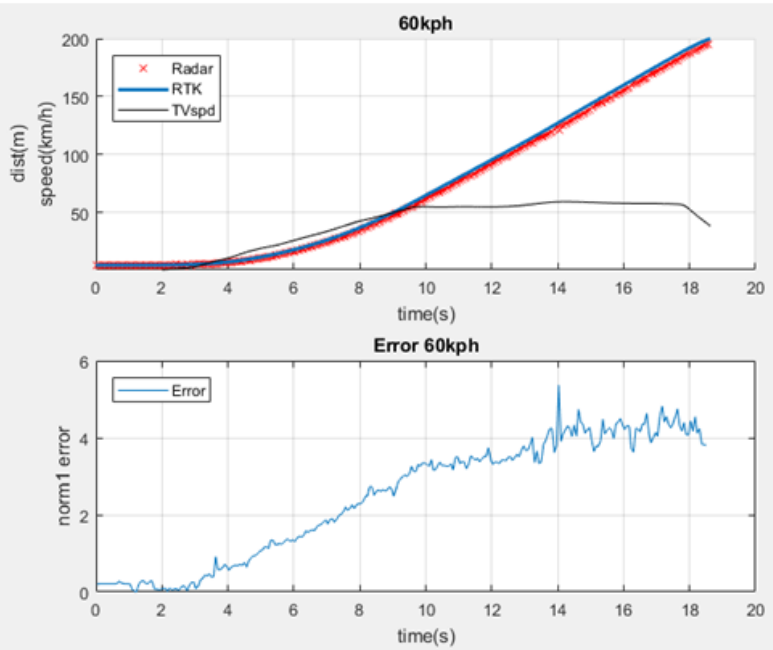

Fig. 5. Data collection of $60 \mathrm{kph}$ 


\section{Detection Time Analysis}

According to Figure 4 and 5, the vehicle speed was positively correlated with the error, which originated from detection time. Therefore, the detection results should accord with the equation of motion:

$$
x_{i}=x_{i-\Delta t}+v_{t-\Delta t} \Delta t+O\left(\Delta t^{2}\right)
$$

where $x$ is the position of the object, $t$ is the current time, and $\Delta t$ is the detection time. Equation 8 can be derived by substituting RTK distance and the Kalman filter observation error model into Equation 7:

$$
\begin{gathered}
\mathbf{x}_{R T \mathrm{x}}=\mathbf{x}_{K a l}+\mathbf{v}_{K a i} \Delta t \\
\mathbf{x}_{R T X}=\left[x_{R T X 1} x_{R T X 2}, \cdots, x_{R T X}\right]^{T} \\
\mathbf{x}_{K a^{\prime}}=\left[x_{K a^{\prime} 1}, x_{K a^{\prime} 2}, \cdots, x_{K a^{\prime} n}\right]^{T} \\
\mathbf{v}_{K a^{\prime}}=\left[v_{X a^{\prime} 1}, v_{X a^{\prime} 2}, \cdots, v_{X a^{\prime} n}\right]^{T}
\end{gathered}
$$

where $x_{R R x_{i}}, x_{K a^{\prime} i z}, v_{K a^{\prime} i}, i \in 1,2, \cdots, n$ denote the RTK distance, Kalman distance, and Kalman velocity for Time $i$ respectively. Because radar and RTK both have detection errors, a solution for $\Delta t$ cannot be obtained directly from Equation 8. Therefore, the least squares method [15] should be used to obtain the optimal $\Delta t$.

$$
\min _{\Delta t}\left(\mathbf{x}_{R T K}-\left(\mathbf{x}_{K a^{\prime}}+\mathbf{v}_{K a^{\prime}} \Delta t\right)_{2}\right)
$$

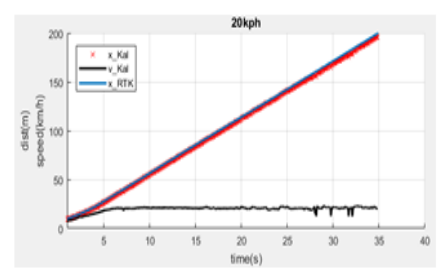

(a) $20 \mathrm{kph}$
Finding an answer for Equation 8 is equivalent to finding an answer for Equation 10.

$$
\min _{\Delta t}\left(\sum_{i=1}^{n}\left(x_{R T_{i}}-\left(x_{K a_{i}^{\prime}}+v_{K a_{i}^{\prime}} \Delta t\right)\right)^{2}\right)
$$

Let $c_{i}=x_{R T_{k}}-x_{K a_{i}}, i \in 1,2, \ldots, n$ and expand Equation 10 to obtain Equation 11:

$$
\min _{\Delta t}\left(\sum_{i=1}^{n}\left(c_{i}^{2}-2 c_{i} v_{K a_{i}^{\prime}} \Delta t+v_{K a_{i}}^{2} \Delta t^{2}\right)\right)
$$

Let $f: \mathrm{R} \rightarrow \mathrm{R}$; Equation 12 is obtained:

$$
f(\Delta t)=\sum_{i=1}^{n}\left(c_{i}^{2}-2 c_{i} v_{K_{a l}} \Delta t+v_{K_{i} l_{i}}^{2} \Delta t^{2}\right)
$$

Finding the minimum value of Equation 9 is equivalent to finding a solution for Equation 13.

$$
\frac{\mathrm{d} f(\Delta t)}{\mathrm{d} \Delta t}=\sum_{i=1}^{n}\left(-2 c_{i} v_{K a l_{i}}+2 v_{K_{a l_{i}}}^{2} \Delta t\right)=0
$$

Subsequently, the analysis results for 20,40 , and $60 \mathrm{kph}$ are input (Figsures $6,7,8$ ) to obtain a solution for $\Delta t$ in Equation 13 Accordingly, Equation 14 can be obtained.

$$
\Delta t \cong 0.2649
$$

Fig. 6. Analysis results

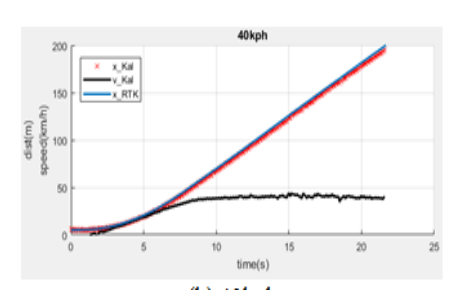

(b) $40 \mathrm{kph}$

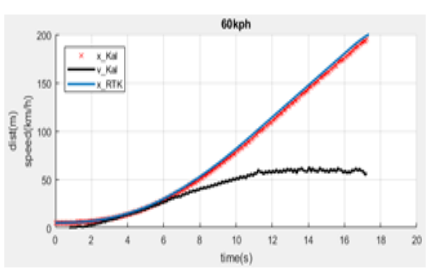

(c) $60 \mathrm{kph}$

\section{Dynamic Modification Method}

By using the aforementioned Kalman filter and observation error model, substituting the results derived from Equation 14 for detection time error analysis into Equation 7 for object motion, and assuming that the current state is $\hat{x}$ and the detection states are $\tilde{x}_{t}$ and $\tilde{v}_{t}$, Equation 15 can be obtained.

$$
\hat{x}_{t}=\tilde{x}_{t}+\tilde{v}_{t} \Delta t
$$

The analysis results are detailed in the next paragraph.

\section{E. Dynamic Modification Results}

In this section, the method introduced in the previous paragraph was used to compare the results of only using the Kalman filter observation error model at a dynamic scene and the results of adding dynamic modification. The millimeter-wave radar was installed at a fixed position and set as the origin. The target vehicle was initially in front of 
the radar (Figure 7 and simultaneously received radar and GPS-RTK information. Figure 8 presents the X-Y cumulative graph for millimeter-wave radar and GPS-RTK. In the figure, the blue line represents RTK-GPS information, red dots represent raw radar data, and green dots represent the outcomes obtained by using the Kalman filter. It is evident that the errors in the horizontal direction were modified after using the proposed method.

Figure 9a presents the graph for 60-kph vertical distance versus time. In the figure, the blue line represents RTK-GPS vertical distance, red dots represent the results of applying the Kalman filter to the radar, and the black line represents the relative speed. The results indicate that errors were observed between radar outcomes and RTK-GPS outcomes. As shown by the blue line in Figure 9a, as the vehicle speed increased to $60 \mathrm{kph}$, the error reached approximately $4 \mathrm{~m}$. The orange line represents the results obtained from dynamic modification. The error was less than $0.5 \mathrm{~m}$ within the range of $100 \mathrm{~m}$; the error did not exceed $1.5 \mathrm{~m}$ within the range of 100-200 m.

Figure $9 \mathrm{~b}$ presents the graph for 40 -kph vertical distance versus time. In the figure, as the vehicle speed increased to $40 \mathrm{kph}$, the error without dynamic modification was approximately $2.5 \mathrm{~m}$. After dynamic modification, the error was less than $0.8 \mathrm{~m}$ within the range of $100 \mathrm{~m}$ and did not exceed $2.2 \mathrm{~m}$ within the range of 100-200. Figure 9c presents the graph for 20 -kph vertical distance versus time. In the figure, as the vehicle speed increased to $20 \mathrm{kph}$, the error was approximately $2 \mathrm{~m}$ without dynamic modification; following dynamic modification, the error was less than 0.6 within the range of $100 \mathrm{~m}$ and did not exceed $2 \mathrm{~m}$ within the range of 100-200 m after deducting four peak values. Fig. 10 presents more results. In the figure, the 100 -m black line represents the time when the target reached $100 \mathrm{~m}$. The results indicated that at the dynamic scene of medium or short distance (within the range of $100 \mathrm{~m}$ ), dynamic modification could effectively reduce the detection errors for vertical distance. For a long distance (100 m or above), radar detection errors (distance times 1.5\%) induced large errors; however, some errors can still be reduced.

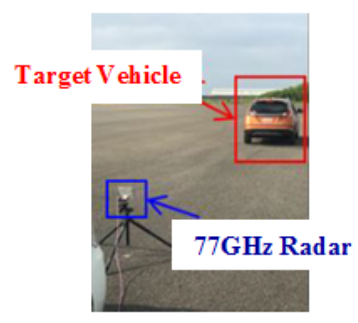

Fig. 7. Analysis results

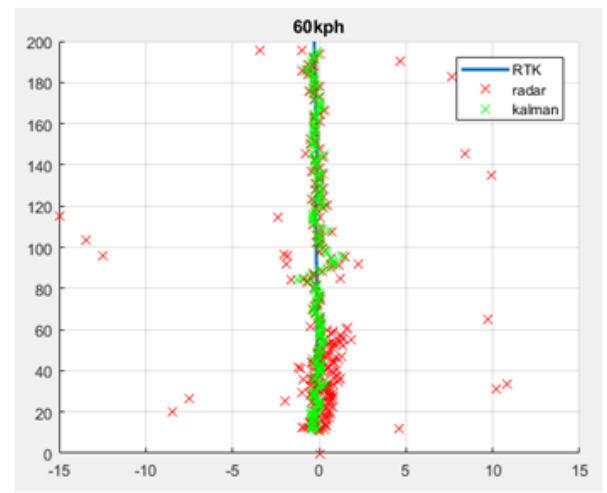

Fig. 8. X-Y cumulative graph of $60 \mathrm{kph}$ dynamic scene

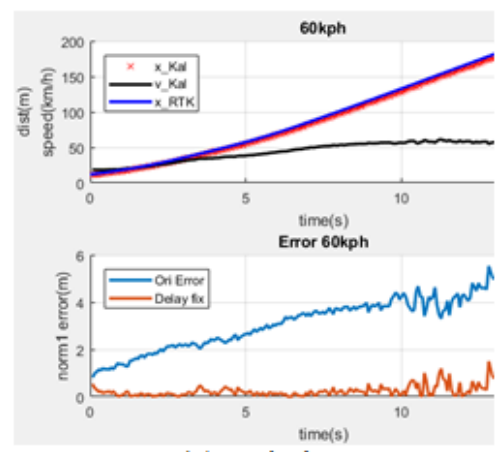

(a) $60 \mathrm{kph}$

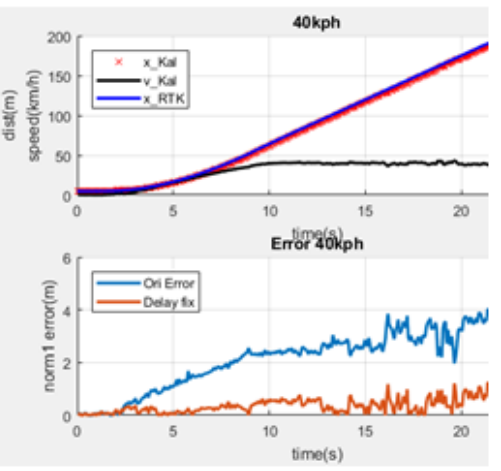

(b) $40 \mathrm{kph}$

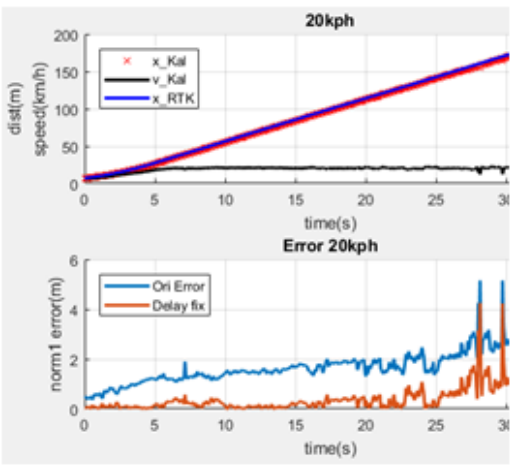

(c) $20 \mathrm{kph}$

Fig. 9. Dynamic scene time to distance $(t-y)$

Table 2 presents the results obtained before and after dynamic modification for various scenes. Before modification, the average error for short, medium, or long distance at the dynamic scene of 20-60 kph was between $1.59 \mathrm{~m}$ (Test 2-Orig. for $20 \mathrm{kph}$ ) and $3.20 \mathrm{~m}$ (Test 2-Orig. for $60 \mathrm{kph}$ ). After modification, the average error was between $0.41 \mathrm{~m}$
(Test 1-Pro. for $60 \mathrm{kph}$ ) and $0.76 \mathrm{~m}$ (Test 2-Pro. for $60 \mathrm{kph}$ ). For a medium or short distance (within the range of $100 \mathrm{~m}$ ), the error was modified from no smaller than $1.07 \mathrm{~m}$ (Test 2-Orig. for $20 \mathrm{kph}$ ) to no greater than 0.52 (Test 2-Pro. for $60 \mathrm{kph})$. 

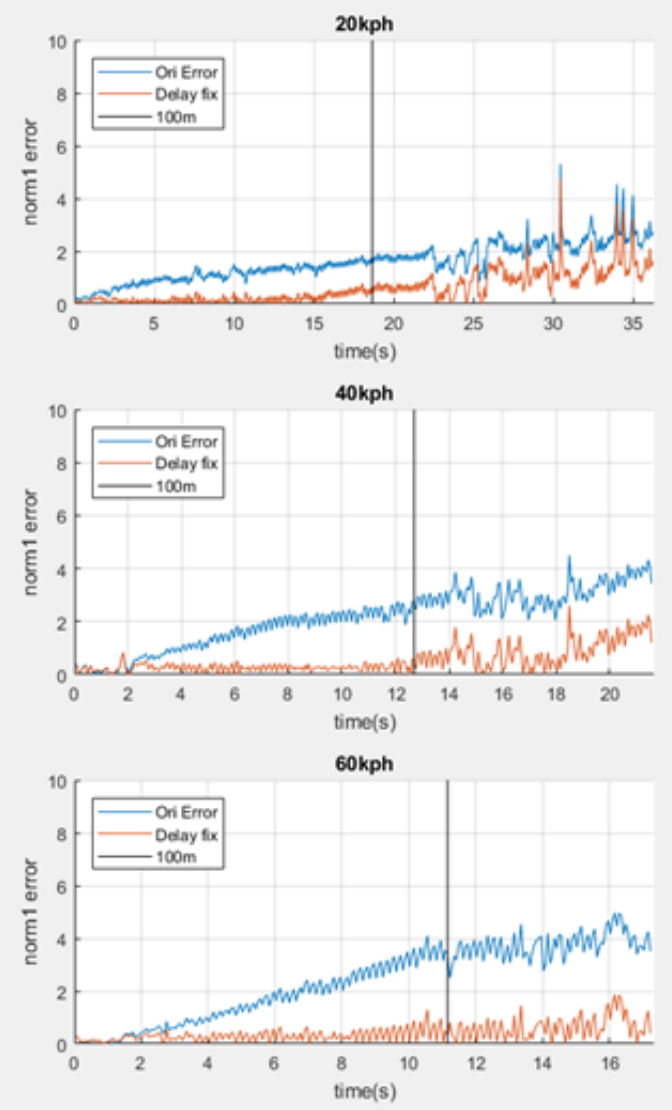
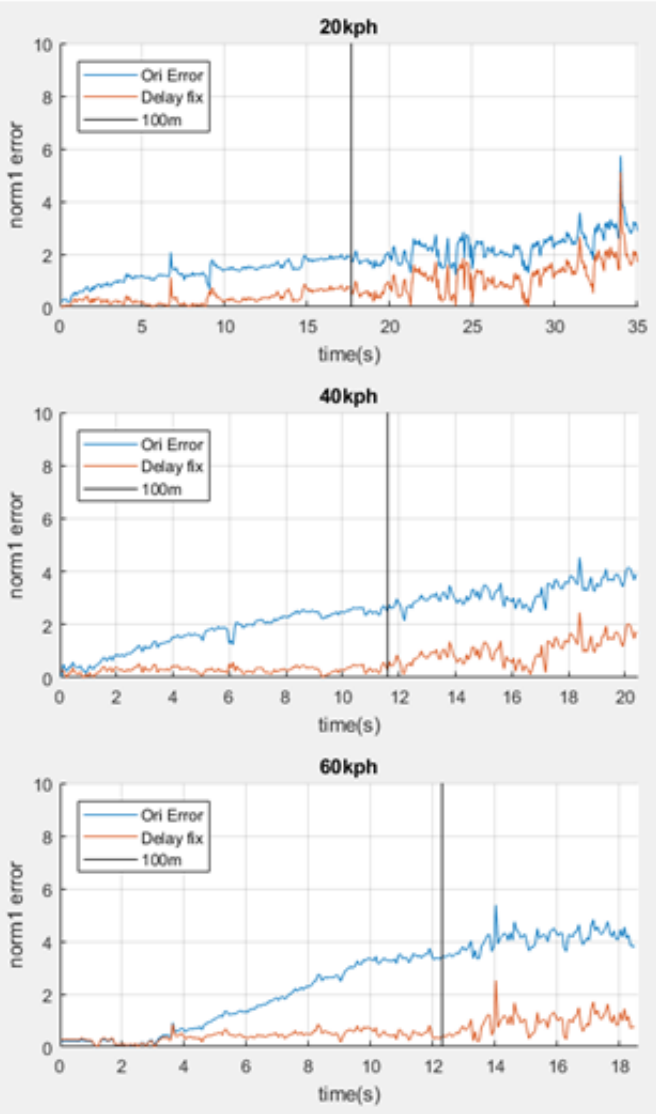

Fig. 10. Experimental results

TABLE 2

COMPARISON WITH THE PROPOSED DYNAMIC CORRECTION RESULTS AND ORIGINAL DATA (METER)

\begin{tabular}{|c|c|c|c|c|c|c|c|c|c|c|c|}
\hline \multirow{3}{*}{$\begin{array}{l}\text { Target } \\
\text { Velocity } \\
\text { (kph) }\end{array}$} & \multirow{3}{*}{$\begin{array}{l}\text { Target } \\
\text { Distance } \\
(m)\end{array}$} & \multirow{2}{*}{\multicolumn{3}{|c|}{$\begin{array}{l}\text { Test } \\
\text { Data } 1\end{array}$}} & \multirow{2}{*}{\multicolumn{3}{|c|}{$\begin{array}{l}\text { Test } \\
\text { Data } 2\end{array}$}} & \multirow{2}{*}{\multicolumn{3}{|c|}{$\begin{array}{l}\text { Test } \\
\text { Data } 3\end{array}$}} & \multirow{3}{*}{$\begin{array}{l}\text { Total } \\
\text { Average } \\
\text { Saving } \\
(\%)\end{array}$} \\
\hline & & & & & & & & & & & \\
\hline & & $\begin{array}{l}\text { Orig. } \\
\text { (m) }\end{array}$ & $\begin{array}{l}\text { Pro. } \\
\text { (m) }\end{array}$ & $\begin{array}{l}\text { Saving } \\
(\%)\end{array}$ & $\begin{array}{l}\text { Orig. } \\
\text { (m) }\end{array}$ & $\begin{array}{l}\text { Pro. } \\
\text { (m) }\end{array}$ & $\begin{array}{l}\text { Saving } \\
(\%)\end{array}$ & $\begin{array}{l}\text { Orig. } \\
\text { (m) }\end{array}$ & $\begin{array}{l}\text { Pro. } \\
\text { (m) }\end{array}$ & $\begin{array}{l}\text { Saving } \\
\text { (\%) }\end{array}$ & \\
\hline \multirow[t]{3}{*}{20} & $<100$ & 1.27 & 0.28 & 77 & 1.07 & 0.20 & 81 & 1.29 & 0.32 & 75 & \multirow[t]{3}{*}{59} \\
\hline & $\geq 100$ & 2.32 & 1.19 & 48 & 2.13 & 1.03 & 51 & 2.27 & 1.13 & 50 & \\
\hline & Avg. & 1.79 & 0.73 & 59 & 1.59 & 0.61 & 61 & 1.77 & 0.72 & 59 & \\
\hline \multirow[t]{3}{*}{40} & $<100$ & 1.46 & 0.26 & 82 & 1.68 & 0.29 & 82 & 1.45 & 0.23 & 84 & \multirow[t]{3}{*}{74} \\
\hline & $\geq 100$ & 3.07 & 0.89 & 71 & 3.20 & 0.99 & 69 & 3.10 & 0.93 & 70 & \\
\hline & Avg. & 2.12 & 0.52 & 75 & 2.34 & 0.59 & 74 & 2.12 & 0.51 & 75 & \\
\hline \multirow[t]{3}{*}{60} & $<100$ & 1.63 & 0.29 & 82 & 2.41 & 0.52 & 78 & 1.63 & 0.40 & 75 & \multirow[t]{3}{*}{78} \\
\hline & $\geq 100$ & 3.75 & 0.62 & 83 & 4.20 & 1.06 & 74 & 4.07 & 0.92 & 77 & \\
\hline & Avg. & 2.37 & 0.41 & 82 & 3.20 & 0.76 & 76 & 2.45 & 0.57 & 76 & \\
\hline
\end{tabular}

\section{CONCLUSION}

The paper presents an innovation dynamic obstacle correction algorithm based on the Kalman filter observation error model. The verification procedures of the proposed algorithm are summarized below:

- Equipment installation:

a. The radar is fixed at tripod, and then this position is set as the original point.

b. The RTK-GPS is installed on the target vehicle.

- Data collection:

a. The data of the above two sensors renders synchronization as necessary.

b. When the subject car forwards to the front, the data collection is enabled until the speed accelerates to a specifi- 
cation speed, and then the speed would be set in a cruise control condition.

- Each radar point information, which includes obstacle distance $\mathbf{x}_{K a^{\prime}}$, and relative speed $\mathbf{v}_{K a l}$, is obtained by the Kalman filter observation error model. Besides, the current distance of obstacle is received based on RTK-GPS.

- Substituting the above information into the following equation:

$$
\Delta t=\left(\left(\mathbf{x}_{\mathrm{RTK}}-\mathbf{x}_{\text {Kal }}\right)^{T} \mathbf{v}_{\text {Kal }}\right) /\left(\mathbf{v}_{\text {Kal }}^{T} \mathbf{v}_{\text {Kal }}\right)
$$

- Substituting $\Delta t$ into the dynamic compensation equation, and then can be obtained:

$$
\hat{x}_{\text {out }}=x_{K a l}+v_{K a l} \Delta t
$$

According to the above procedures, steps 1 to 4 are mainly responsible for collecting and analyzing the input data. On the actual application, step 5 which is lower complexity computation can be directly used to compute the dynamic compensation results. From experimental results, it's shown that the proposed method can achieve a stable detection and effectively reduce the detection error of dynamic scene, which is achieved as 59\%, 74\%, and 78\% improving in $20 \mathrm{kph}, 40 \mathrm{kph}$, and $60 \mathrm{kph}$ relative speed when comparing with the original data.

\section{ACKNOWLEDGMENT}

This study is part of the results of the TDP for the Nonprofit Research Organization plan (No. 108- EC-17-A-25-1379) of the Automotive Research \& Testing Center (ARTC).

\section{REFERENCES}

[1] A. Paul, R. Chauhan, R. Srivastava, and M. Baruah, "Advanced driver assistance systems 2016-28-0223," in International Mobility Conference, Bangkok, Thailand, 2016.

[2] L. Zhou, "A new minimal solution for the extrinsic calibration of a 2D LIDAR and a camera using three plane-line correspondences," IEEE Sensors Journal, vol. 14, no. 2, pp. 442-454, 2013. doi: https://doi.org/10.1109/jsen.2013.2284789

[3] S. Yang, H.-S. Lho, and B. Song, "Sensor fusion for obstacle detection and its application to an unmanned ground vehicle," in International Joint Conference, Rome, Italy, 2009.

[4] V. Ilic, M. Marijan, A. Mehmed, and M. Antlanger, "Development of sensor fusion based adas modules in virtual environments," in Zooming Innovation in Consumer Technologies Conference, California, CA, 2018.

[5] H. Cho, Y.-W. Seo, B. V. Kumar, and R. R. Rajkumar, "A multi-sensor fusion system for moving object detection and tracking in urban driving environments," in IEEE International Conference on Robotics and Automation, New Dehli, India, 2014.

[6] J. Kim, D. S. Han, and B. Senouci, "Radar and vision sensor fusion for object detection in autonomous vehicle surroundings," in Tenth International Conference on Ubiquitous and Future Networks, Porto, Portugal, 2018.

[7] S. Durand, R. Benmokhtar, and X. Perrotton, "360 multisensor object fusion and sensor-based erroneous data management for autonomous vehicles," in IEEE Sensors Applications Symposium, Sophia Antipolis, France, 2019.

[8] M.-S. Hsieh, S.-Y. Luo, P.-H. Liao, and D.-Q. Ye, "Implementation of dynamic boundary on multiple kalman trackings using radar," in IEEE 20th International Conference on Intelligent Transportation Systems, Yokohama, Japan, 2017.

[9] J. Kocić, N. Jovičić, and V. Drndarević, "Sensors and sensor fusion in autonomous vehicles,' ' in 26th Telecommunications Forum, Belgrade, Serbia, 2018.

[10] A. Mecocci, M. Shahar, P. Ericsson, S. Piccand, I. Ravyse, T. Llewellyn, and D. Di Censo, "Sensors fusion paradigm for smart interactions between driver and vehicle," in IEEE SEensor Conference, New York, NY, 2016.

[11] Z. Ji and D. Prokhorov, "Radar-vision fusion for object classification," in 11th International Conference on Information Fusion,Cologne, Germany, 2008.

[12] S. R. Axelsson, "Position correction of moving targets in SAR imagery," in SAR Image Analysis, Modeling, and Techniques, Dresden, Germany, 2004.

[13] S. Wittayapiyanon and S. Chuepeng, "Sports utility vehicle seat structure simulation of frontal oblique crash," Journal of Advances in Technology and Engineering Research, vol. 3, no. 3, pp. 69-78, 2017. doi: https://doi.org/10.20474/jater-3. 3.2

[14] H. Khadija and K. Elif, "Contact less signs monitoring using doppler radar sensor," Journal of Advances in Technology and Engineering Research, vol. 5, no. 2, pp. 72-78, 2019. doi: https://doi.org/10.20474/jater-5.2.2

[15] L. Po-Hsiang, "Kalman filter observation error model applied to radar sensing dynamic vehicle tracking," in National Conference on Vehicle Engineering, New York, NY, 2017. 
[16] M. Attom, M. Kou, and N. Al-Akhras, "Geo environmental utilization of iron-filing with cement in soil stabilization," International Journal of Technology and Engineering Studies, vol. 2, no. 2, pp. 32-37, 2016. doi: https://doi.org/10. 20469/ijtes.2.40001-2

[17] R. E. Kalman, "A new approach to linear filtering and prediction problems," Journal of Basic Engineering, vol. 82, no. 1, pp. 35-45, 1960. doi: https://doi.org/10.1115/1.3662552

[18] E. Uma and A. Kannan, "Self-aware message validating algorithm for preventing xml-based injection attacks," International Journal of Technology and Engineering Studies, vol. 2, no. 3, pp. 60-69, 2016. doi: https://doi.org/10.20469/ijtes. 2.40001-3

[19] H. Moritz, “Advanced least-squares methods," Dept of Geodetic Science, Ohio State University, Ohio, OI, Technical report, 1972 . 